\title{
Constituintes químicos, avaliação das atividades citotóxica e antioxidante de Mimosa paraibana Barneby (Mimosaceae)
}

\author{
Xirley P. Nunes, ${ }^{* 1,2}$ Rafael F. Mesquita, ${ }^{1}$ Davi A. Silva, ${ }^{2}$ Daysianne P. Lira, ${ }^{2}$ Vicente C. O. \\ Costa, ${ }^{2}$ Marianna V. B. Silva, ${ }^{2}$ Aline L. Xavier, ${ }^{2}$ Margareth F. F. M. Diniz, ${ }^{2}$ \\ Maria de Fátima Agra $^{2}$ \\ ${ }^{1}$ Colegiado de Medicina, Universidade Federal do Vale do São Francisco, Caixa Postal 252, \\ 56306-205 Petrolina-PE, Brasil, \\ ${ }^{2}$ Laboratório de Tecnologia Farmacêutica, Universidade Federal da Paraíba, Caixa Postal 5009, 58051-970 \\ João Pessoa-PB, Brasil
}

\begin{abstract}
RESUMO: Mimosa paraibana Barneby foi submetida a um estudo fitoquímico para o isolamento de seus constituintes químicos, através de métodos cromatográficos usuais, e posterior identificação estrutural, utilizando-se métodos espectroscópicos de $\mathrm{RMN}{ }^{1} \mathrm{H} \mathrm{e}{ }^{13} \mathrm{C}$ uni e bidimensionais, além de comparações com modelos da literatura. Deste estudo pioneiro, foram isolados e identificados cinco constituintes da fase clorofórmica: uma mistura dos esteróides, $\beta$-sitosterol e estigmasterol, a $15^{1}$-hidroxi-feofitina A, a 5,7-dihidroxiflavanona, o 3,4,5-trihidroxibenzoato de etila e o ácido p-cumárico. A atividade antioxidante das fases hexânica, clorofórmica e acetato de etila foi avaliada utilizando o radical estável DPPH (1,1-difenil-2-picril-hidrazil) e os resultados comparados com o padrão ácido ascórbico. A avaliação da citotoxicidade das fases foi realizada empregando-se o ensaio de letalidade contra Artemia salina. Dos extratos avaliados, somente o hexânico mostrou baixa toxicidade
\end{abstract}

Unitermos: Mimosa paraibana, Mimosaceae, Artemia salina, atividade antioxidante.

\begin{abstract}
Chemical constituents, evaluation of the cytotoxic and antioxidant activities of Mimosa paraibana Barneby (Mimosaceae)”. The phytochemical study of Mimosa paraibana Barneby led to the isolation of its chemical constituents, through the usual chromatographic methods, and further structural identification, using ${ }^{1} \mathrm{H}$ and ${ }^{13} \mathrm{C}$ NMR spectroscopic methods based on one and two-dimensional techniques, in addition to comparison with literature data. From this pioneering investigation with $M$. paraibana, five constituents were isolated and identified from the chloroform extract: a mixture of $\beta$-sitosterol and stigmasterol, $15^{1}$-hydroxy-phaeophytin A, 5,7-dihydroxyflavanone, ethyl 3,4,5-trihydroxybenzoate and $p$-coumaric acid. The antioxidant activity of the hexane, chloroform and ethyl acetate extracts of $M$. paraibana were measured using the 1,1-diphenyl-2-picryl-hydrazyl (DPPH) free radical scavenging assay and the results compared with standard ascorbic acid. The toxicity activity of the extracts were performed using the bioassay of Artemia salina.
\end{abstract}

Keywords: Mimosa paraibana, Mimosaceae, Artemia salina, antioxidant activity.

\section{INTRODUÇÃO}

A família Mimosaceae possui cerca de 60 gêneros distribuídos em mais de 4000 espécies encontradas nas regiões tropicais e subtropicais, especialmente nas regiões áridas. As plantas desta família são principalmente arbustos que apresentam suas folhas verdes praticamente o ano inteiro e seus frutos são geralmente legume. Mimosa é um gênero de leguminosas pantropical, constando de cerca de 480 espécies, com uma maior concentração de espécies na América do Sul (Neilson, 1992). Muitas espécies desse gênero são utilizadas na medicina popular (Agra et al., 2007 \& 2008) e fonte de alcalóides, flavonóides, terpenóides e carotenóides (Barbosa-Filho et al., 2008; ISI-Plataforma
Web of knowledge 2008).

Mimosa paraibana Barneby é encontrada em vários lugares do estado da Paraíba e popularmente conhecida como "jurema”. Este estudo relata o primeiro estudo químico e farmacológico envolvendo esta espécie.

Desde o início da vida no planeta, a toxicidade do oxigênio atmosférico tem sido o principal desafio à sobrevivência dos organismos vivos. As oxidações biológicas geram espécies reativas de oxigênio (ERO) e radicais livres que podem provocar lesões celulares, muitas vezes irreparáveis (Gouvêa, 2004).

As plantas produzem uma grande variedade de substâncias antioxidantes contra os danos moleculares causadosporERO.Oscompostosfenólicoscompreendem 
o principal grupo de compostos antioxidantes de origem vegetal. Entre os fenóis, os flavonóides constituem o grupo mais importante. Os antioxidantes naturais são bem indicados para atenuar os efeitos deletérios do estresse oxidativo celular. Estudos recentes mostram que vários extratos de plantas exercem ação antioxidante. A partir da perspectiva do valor potencial dos antioxidantes, as pesquisas buscam extratos e substâncias naturais com potente atividade antioxidante e baixa citotoxicidade (Gouvêa, 2004; Lima et al., 2006; Andrade et al., 2007; Rocha et al., 2007; Souza et al., 2007; Vicentino \& Menezes, 2007; Balestrin et al., 2008; Iha et al., 2008; Rodríguez et al., 2008).

A fim de estabelecer a toxicidade de novos produtos naturais, muitos ensaios podem ser utilizados, como o ensaio de letalidade com o microcrustáceo Artemia salina, que foi desenvolvido para detectar compostos bioativos em extratos vegetais (Meyer et al., 1982; Nick et al., 1995; Ruiz et al., 2005; Lhullier et al., 2006; Silva et al., 2007), mas que também pode ser utilizado para expressar a toxicidade de produtos naturais, como extratos de plantas e de produtos marinhos (Shoeb et al., 2007; Mota et al., 2008). Adicionalmente, alguns trabalhos mostram uma boa correlação entre o ensaio de letalidade com larvas de A. salina e a citotoxicidade em linhagens de células humanas para esses produtos (McLaughlin et al., 1995; Carballo et al., 2002).

A avaliação da bioatividade de extratos de plantas, medida pela toxicidade frente Artemia salina, pode fornecer informações valiosas ao trabalho de químicos de produtos naturais e farmacólogos, indicando fontes vegetais com importantes atividades biológicas. Neste contexto, a utilização de bioensaios para o monitoramento da bioatividade de extratos, frações e compostos isolados de plantas vem crescendo consideravelmente nos laboratórios de pesquisa em nível mundial como método alternativo para o uso de animais de laboratório. Artemia salina Leach é um microcrustáceo amplamente conhecido como indicador de toxicidade em um bioensaio que utiliza a $\mathrm{CL}_{50}$ (concentração letal média) como parâmetro de avaliação da atividade biológica.

Este trabalho descreve os metabólitos secundários da fase clorofórmica de M. paraibana, a avaliação da citotoxicidade do extrato etanólico bruto e fases sobre Artemia salina Leach e a atividade antioxidante das fases através da metodologia do radical livre estável $\mathrm{DPPH}$.

\section{MATERIAL E MÉTODOS}

\section{Equipamentos}

Os espectros de RMN $\left({ }^{1} \mathrm{H},{ }^{13} \mathrm{C}, \mathrm{HMQC}, \mathrm{HMBC}\right.$, COSY, NOESY) foram obtidos em espectrômetro Mercury Varian operando a $200 \mathrm{MHz}\left({ }^{1} \mathrm{H}\right)$ e $50 \mathrm{MHz}$ $\left({ }^{13} \mathrm{C}\right)$, no Laboratório de Tecnologia Farmacêutica (LTF), da Universidade Federal da Paraíba (UFPB). Os dados de RMN foram mensurados em $\mathrm{CDCl}_{3}$ ou $\mathrm{CD}_{3} \mathrm{OD}$ e os deslocamentos químicos foram expressos em ppm com o sinal do solvente como referência.

\section{Material vegetal}

As partes aéreas de Mimosa paraibana Barneby foram coletadas no município de Serra Branca, estado da Paraíba, em abril de 2005. O material botânico foi identificado pela Prof. Dra. Maria de Fátima Agra. Uma exsicata da espécie está depositada no Herbário do Laboratório de Tecnologia Farmacêutica (LTF), da Universidade Federal da Paraíba (UFPB) sob o código Agra et al. 6460.

\section{Extração e isolamento}

O material vegetal foi seco em estufa com ar circulante à temperatura média de $40{ }^{\circ} \mathrm{C}$ durante 4 dias. Após a secagem, foi submetido a um processo de pulverização em moinho mecânico, obtendo-se 8 kg de pó. Em seguida o pó foi submetido à maceração exaustiva com etanol (EtOH) a $95 \%$. A solução etanólica obtida foi filtrada e evaporada com o auxílio de um rotaevaporador a uma temperatura média de 50 ${ }^{\circ} \mathrm{C}$, obtendo-se $829 \mathrm{~g}$ de extrato etanólico bruto (EEB). $\mathrm{O}$ EEB foi solubilizado numa mistura $\mathrm{MeOH}: \mathrm{H}_{2} \mathrm{O}$ (3:7 v/v) sob agitação mecânica, obtendo-se uma solução hidroalcoólica I, que foi submetida a uma extração líquido/líquido com solventes de polaridade crescente fornecendo as fases hexânica, clorofórmica e acetato de etila.

A fase clorofórmica (159 g) foi submetida à cromatografia em coluna, utilizando sílica gel 60, como fase estacionária, e como eluentes, os solventes hexano, clorofórmio e metanol puros ou em misturas binárias, em grau crescente de polaridade. As frações foram concentradas em rotaevaporador rotatório e reunidas após cromatografia em camada delgada analítica. As frações 25/29 foram reunidas e recristalizadas em hexano, resultando em cristais brancos (1) (47 mg). As frações 64/91 foram reunidas e submetidas à cromatografia em camada delgada preparativa (CCPD), resultando em um sólido amorfo verde (2) (41 mg). As frações 137/163 foram reunidas e cromatografadas em coluna de Sephadex LH-20 e eluídas com misturas binárias de clorofórmio e metanol, obtendo-se um pó fino amarelo escuro (3) (38 mg). As frações 180/198 e 199/212 foram reunidas e submetidas à CCDP, resultando em um sólido branco (4) (74 mg) e um sólido amorfo branco (5) (83 $\mathrm{mg})$, respectivamente.

\section{Ensaio de letalidade com Artemia salina}

A atividade citotóxica do extrato etanólico bruto e das fases hexânica, clorofórmica e acetato de 
etila de $M$. paraibana foi avaliada através do teste de letalidade contra Artemia salina Leach, de acordo com o método proposto por Meyer et al. (1982), com algumas modificações. Ovos de Artemia salina foram incubados em água do mar artificial a temperatura ambiente por 48 horas. Com a ajuda de uma fonte de luz, as larvas foram atraídas e coletadas. Soluções do extrato e fases foram preparadas em Tween 80 ou DMSO (Dimetil sulfóxido) e água do mar em concentrações variando de 20-1000 $\mu \mathrm{g} / \mathrm{mL}$. Cerca de 13 a 15 metanáuplios foram transferidos para tubos de ensaio contendo $5 \mathrm{~mL}$ de cada uma das soluções a serem testadas. Um grupo controle foi preparado contendo apenas os solventes e as larvas. Os ensaios foram realizados em triplicata. A contagem do número de larvas mortas foi realizada após 24 horas e esse número foi usado para o cálculo da $\mathrm{CL}_{50}$ utilizandose o programa Microcal Origin versão 4.1. Os extratos com $\mathrm{CL}_{50}$ maiores que $1000 \mathrm{ppm}$ foram considerados inativos.

\section{Dosagem da atividade antioxidante}

A dosagem de atividade antioxidante das fases hexânica, clorofórmica e acetato de etila de M. paraibana foi realizada pelo método fotocolorimétrico in vitro do radical livre estável DPPH (1,1-difenil-2-picril-hidrazil) obtido da SIGMA. Nesse método é preparada uma solução de $45 \mu \mathrm{g} / \mathrm{mL}$ de DPPH em metanol. As amostras são preparadas adicionando-se $1 \mathrm{~mL}$ desta solução de DPPH a 2,5 mL de soluções das fases diluídas em metanol a concentrações de 5, 10, 25, 50, 125 e $250 \mu \mathrm{g} /$ $\mathrm{mL}$. Como branco foi utilizado $1 \mathrm{~mL}$ de DPPH e 2,5 mL de metanol. Como controle positivo foi utilizado o ácido L-(+)-ascórbico (Merck).

A solução de DPPH possui uma coloração roxa intensa e a ação antioxidante de um extrato ou fase pode ser visualizada pelo progressivo descoloramento da solução, ao final do qual a mesma torna-se amarelada. Quinze minutos após a adição de DPPH às amostras, é<smiles>CCC(CC[C@@H](C)C1CCC2C3CC=C4CC(O)CCC4(C)C3CCC21C)C(C)C</smiles>

1<smiles>CCC(/C=C/[C@H](C)C1CCC2C3CC=C4CC(O)CCC4(C)C3CCC21C)C(C)C</smiles>
2

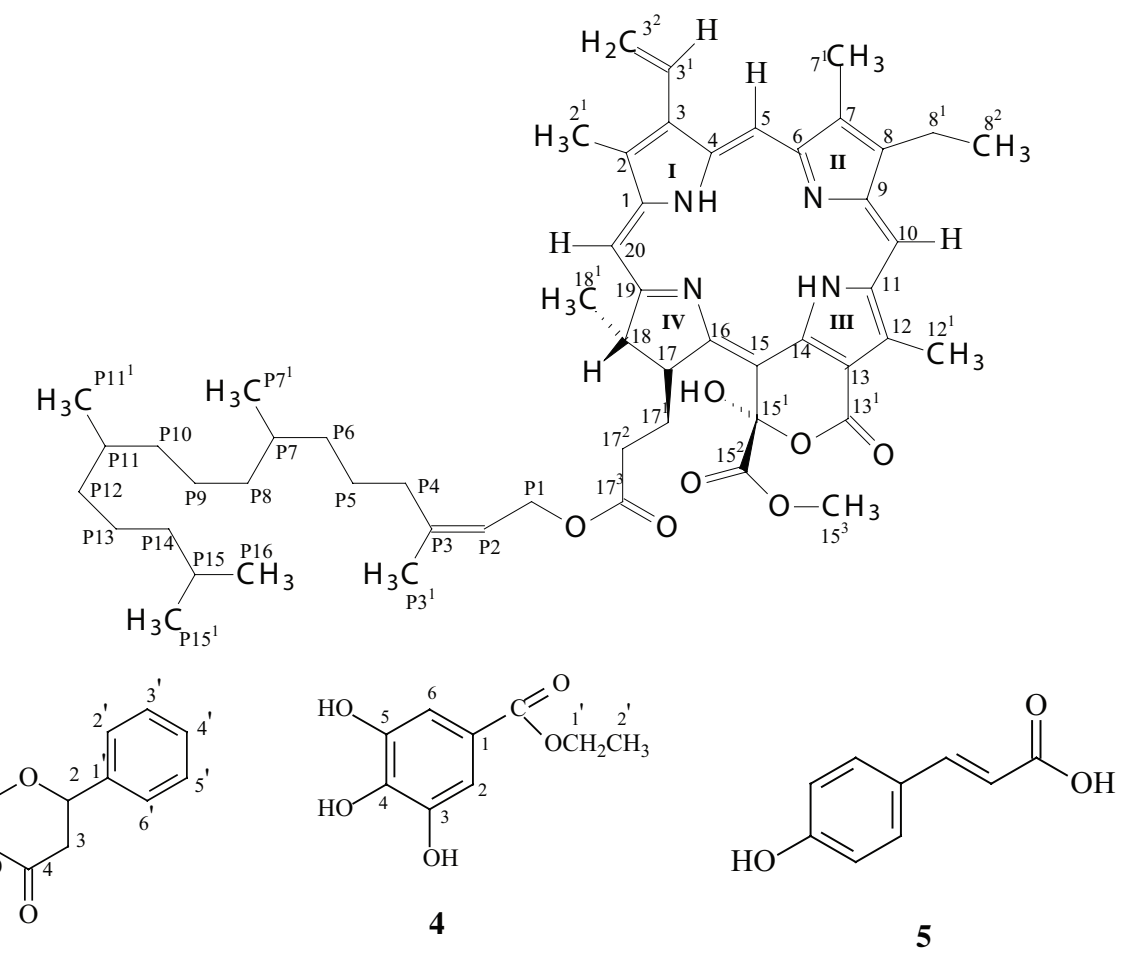

Figura 1. Constituintes químicos isolados da fase clorofórmica de Mimosa paraibana. 


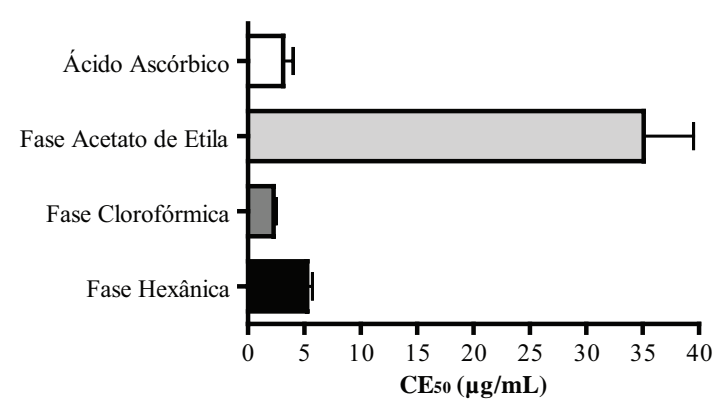

Figura 2. Comparação dos valores de CE50 das diferentes fases obtidas de Mimosa paraibana. Os resultados são expressos como a média \pm SEM $(n=3)$.

feita a leitura em um espectrofotômetro de Ultravioleta UV-Vis Shimadzu UV 1601 em 517 nm. Todas as leituras foram realizadas em triplicata e, com a média dos dados obtidos foi calculada a diferença de absorbância entre a amostra e o controle negativo e as atividades antioxidantes percentuais foram obtidas por regressão linear, logarítimo ou exponencial para cada fase, chegando-se assim à concentração necessária para se obter $50 \%$ do efeito antioxidante máximo estimado de $100 \%\left(\mathrm{CE}_{50}\right.$ ) (Mensor et al., 2001).

As atividades seqüestrantes de DPPH das fases foram expressas em porcentagem, segundo a equação:

Atividade seqüestrante de DPPH

$(\%)=100-[(\underline{A c-A t}) \times 100]$

Ac

Onde, Ac é a absorbância do controle, que não foi incubado com as fases e At a absorbância das amostras incubadas com diferentes concentrações das fases.

\section{RESULTADOS E DISCUSSÃO}

A análise dos dados espectrais e comparação com valores da literatura permitiram identificar a substância 1 como sendo uma mistura dos esteróides $\beta$-sitosterol e estigmasterol (Kojima et al., 1990), a substância 2 como a 151-hidroxi-feofitina A, a substância 3 como a 5,7-dihidroxiflavanona, a substância 4 como o 3,4,5trihidroxibenzoato de etila e a substância 5 como o ácido p-cumárico (Fig. 1) (Mihara et al., 2004).
15-Hidroxi-feofitina A (2): RMN ${ }^{1} \mathrm{H}(200 \mathrm{MHz}$, $\left.\mathrm{CDCl}_{3}\right) \delta_{\mathrm{H}}\left(\mathrm{H}\right.$; mult.; $J$ em Hz): 7,98 $\left(\mathrm{H}-3^{1}\right.$; $d d, 11,2$ e 17,6), 9,48 (H-5; s), 9,71 (H-10; s), 4,07 (H-17; m), 5,13 (P2; $t, 7,4), 8,70(\mathrm{H}-20 ; s), 6,30\left(\mathrm{H}-3^{2} ; d ; 17,8\right)$, 6,15 (H-32 ; d; 11,4), 3,69 (H-8 $;$; , 8,0 e 15,6), 3,42 ( $\left.2^{1} ; s\right), 3,22\left(7^{1} ; s\right), 1,68\left(8^{2} ; t ; 7,6\right), 3,87\left(12^{1} ; s\right), 3,75$ $\left(15^{3} ; s\right), 1,61\left(18^{1} ; t ; 7,2\right) . \mathrm{RMN}{ }^{13} \mathrm{C}\left(50 \mathrm{MHz}, \mathrm{CDCl}_{3}\right)$ $\delta_{\mathrm{C}}: 141,18$ (C-1), 131,45 (C-2), 136,46 (C-3), 135,98 (C-4), 155,71 (C-6), 134,75 (C-7), 145,51 (C-8), 149, 49 (C-9), 138,70 (C-11), 131,45 (C-12), 131,45 (C-13), 166,30 (C-13²), 149,94 (C-14), 111,29 (C-15), 101,95 (C-15'), 170,87 (C-15²), 161,06 (C-16), 171,30 (C-17³), 171,12 (C-19), 142,86 (C-P3), 128,93 (C-3 $\left.{ }^{1}\right), 99,59$ (C-5), 104,10 (C-10), 53,68 (C-17), 50,12 (C-18), 93,87 (C-20), 117,70 (C- P2), 122,71 (C-32), 19,51 (C-81), 29,69 (C-17'), 31,29 (C-172), 61,45 (C-P1), 12,13 (C-21), 11,24 (C-71), 17,58 (C-8²), 12,43 (C-12'), 54,15 $\left(\mathrm{C}-15^{3}\right), 22,70\left(\mathrm{C}-18^{1}\right)$. Todos os valores estão de acordo com os dados da literatura (Ma \& Dolphin, 1996).

5,7-Dihidroxiflavanona (3). RMN ${ }^{1} \mathrm{H}(200 \mathrm{MHz}$, $\left.\mathrm{CDCl}_{3}\right) \delta_{\mathrm{H}}(\mathrm{H}$; mult.; $J$ em Hz): 5,40 (H-2; $d d ; 12,6$ e 3,2), 5,99 (H-6; sl), 5,99 (H-8; sl), 7,44-7,38 (H-2'; m), 7,44-7,38 (H-3'; m), 7,44-7,38 (H-4'; $m$ ), 7,44-7,38 (H-5'; m), 7,44-7,38 (H-6’; m), 3,07 (H-3; dd; 12,6 е 17), 2,79 (H-3; dd; 3,2 e 17). RMN ${ }^{13} \mathrm{C}\left(50 \mathrm{MHz}, \mathrm{CDCl}_{3}\right)$ $\delta_{\mathrm{C}}: 195,81$ (C-4), 164,29 (C-5), 164,72 (C-7), 163,14 (C-9), 103,14 (C-10), 138,24 (C-1'), 79,21 (C-2), 96,75 (C-6), 95,48 (C-8), 126,14 (C-2'), 128,88 (C-3'), 128,88 (C-4'), 128,88 (C-5'), 126,14 (6'), 43,30 (C-3). Todos os valores estão de acordo com os dados da literatura (Agrawal, 1989).

3,4,5-Trihidroxibenzoato de etila (4). $\mathrm{RMN}{ }^{1} \mathrm{H}$ $\left(200 \mathrm{MHz}, \mathrm{CDCl}_{3}\right) \delta_{\mathrm{H}}(\mathrm{H}$; mult.; $J$ em Hz): 7,04 (H-2; s), 7,04 (H-6; s), 4,23 (H-1'; q), 1,30 (H-2'; t). RMN ${ }^{13} \mathrm{C}\left(50 \mathrm{MHz}, \mathrm{CDCl}_{3}\right) \delta_{\mathrm{C}}: 121,63(\mathrm{C}-1), 146,33(\mathrm{C}-3)$, 139,56 (C-4), 146,33 (C-5), 109,93 (C-2), 109,33 (C-6), 61,66 (C-1'), 14,55 (C-2'), 168,51 (CO). Todos os valores estão de acordo com os dados da literatura (SDBS Information, 2008).

No ensaio de toxicidade com Artemia salina os valores médios de $\mathrm{CL}_{50}$ para o extrato etanólico e fases hexânica, clorofórmica e acetato de etila de $M$. paraibana foram de 602,5 $\mu \mathrm{g} / \mathrm{mL}, 1054,0 \mu \mathrm{g} / \mathrm{mL}, 778,8$ $\mu \mathrm{g} / \mathrm{mL}$ e $293,4 \mu \mathrm{g} / \mathrm{mL}$, respectivamente. Portanto, das

Tabela 1. Atividade antioxidante das diferentes fases de Mimosa paraibana utilizando o radical DPPH.

\begin{tabular}{ccccc}
\hline[]$\mu \mathrm{g} / \mathrm{mL}$ & Fase Hexânica & Fase Clorofórmica & Fase Acetato de Etila & Ácido Ascórbico \\
\hline 5 & $26,036 \pm 3,830$ & $12,633 \pm 2,126$ & $66,379 \pm 4,256$ & $71,855 \pm 3,737$ \\
10 & $35,447 \pm 2,465$ & $22,697 \pm 2,215$ & $65,872 \pm 4,287$ & $78,082 \pm 1,678$ \\
25 & $58,555 \pm 2,198$ & $53,274 \pm 4,186$ & $72,709 \pm 2,865$ & $92,123 \pm 1,077$ \\
50 & $73,068 \pm 1,449$ & $57,248 \pm 3,510$ & $77,400 \pm 2,766$ & $93,796 \pm 0,744$ \\
125 & $71,334 \pm 1,231$ & $54,073 \pm 3,893$ & $85,062 \pm 1,791$ & $93,711 \pm 1,133$ \\
250 & $67,108 \pm 1,990$ & $43,988 \pm 4,278$ & $83,817 \pm 1,593$ & $94,424 \pm 0,698$ \\
$\mathrm{CE}_{50}$ & 5,214 & 1,014 & 35,78 & 2,910 \\
\hline
\end{tabular}


amostras testadas, apenas a fase hexânica apresentou baixa toxicidade $\left(\mathrm{CL}_{50}>1000 \mu \mathrm{g} / \mathrm{mL}\right)$. A toxicidade com A. salina mostra boa correlação com atividades antitumoral, inseticida (Meyer et al., 1982; Mclaughlin et al., 1995) e anti-Trypanosoma cruzi (Alves et al., 2000) para substâncias com $\mathrm{CL}_{50}<1000 \mu \mathrm{g} / \mathrm{mL}$. Por outro lado, uma baixa toxicidade pode ser considerada uma característica interessante para utilização de extratos vegetais em ambientes naturais, por exemplo, para o controle da população de caramujos.

A análise dos resultados da atividade antioxidante considera como valor de referência a $\mathrm{CE}_{50}$ do ácido ascórbico $(2,910 \mu \mathrm{g} / \mathrm{mL})$ para comparar a atividade antioxidante das fases testadas. Foi escolhido o ácido ascórbico como parâmetro porque ele possui alta atividade antioxidante (Mensor et al., 2001).

A partir dos resultados apresentados na Figura 2 e Tabela 1 pode-se observar que a fase clorofórmica, quando comparada com o ácido ascórbico, possui uma $\mathrm{CE}_{50}$ igual a $1,014 \mu \mathrm{g} / \mathrm{mL}$, indicando uma atividade antioxidante (AAO) cerca de 2,87 vezes maior do que a apresentada pelo padrão ácido ascórbico. Esse fato se deve provavelmente às substâncias fenólicas presentes nessa fase.

\section{AGRADECIMENTOS}

Os autores agradecem a CAPES, CNPq e RENORBIO/IMSEAR pelo apoio finaceiro.

\section{REFERÊNCIAS}

Agra MF, França PF, Barbosa-Filho JM 2007. Synopsis of the plants known as medicinal and poisonous in Northeast of Brazil. Rev Bras Farmacogn 17: 114140.

Agra MF, Silva KN, Basílio IJLD, França PF, Barbosa-Filho JM 2008. Survey of medicinal plants used in the region Northeast of Brazil. Rev Bras Farmacogn 18: 472-508.

Agrawal PK 1989. Carbon-13 NMR of Flavonoids: Studies in Organic Chemistry 39. Lucknov, India: Elsevier.

Alves TMD, Silva AF, Brandão M, Grandi TSM, Smânia EFA, Smânia A, Zani CL 2000. Biological screening of Brazilian medicinal plants. Mem Inst Oswaldo Cruz 95: 367-373.

Andrade CA, Costa CK, Bora K, Miguel MD, Miguel OG, Kerber VA 2007. Determinação do conteúdo fenólico e avaliação da atividade antioxidante de Acacia podalyriifolia A. Cunn. ex G. Don, Leguminosaemimosoideae. Rev Bras Farmacogn 17: 231-235.

Balestrin L, Dias JFG, Miguel OG, Dall'Stella DSG, Miguel MD 2008. Contribuição ao estudo fitoquímico de Dorstenia multiformis Miquel (Moraceae) com abordagem em atividade antioxidante. Rev Bras Farmacogn 18: 230-235.

Barbosa-Filho JM, Alencar AA, Nunes XP, Tomaz ACA, Sena-Filho JG, Athayde-Filho PF, Silva MS, Souza MFV, da-Cunha EVL 2008. Sources of alpha-, beta-, gamma-, delta- and epsilon-carotenes: A twentieth century review. Rev Bras Farmacogn 18: 135-154.

Carballo JL, Hernández-Inda ZL, Pérez P, García-Grávalos MD 2002. A comparison between two brine shrimp assays to detect in vitro cytotoxicity in marine natural products. BMC Biotechnol 2: 1-5.

Gouvêa CMCP 2004. Oxidações biológicas e atividade vegetal. In: Fitoterápicos anti-inflamatórios: aspectos químicos, farmacológicos e aplicações terapêuticas. Carvalho, J.C.T. coord. Ribeirão Preto: Tecmedd, p. 101-124.

Iha SM, Migliato KF, Vellosa JCR, Sacramento LVS, Pietro RCLR, Isaac VLB, Brunetti IL, Corrêa MA, Salgado HRN 2008. Estudo fitoquímico de goiaba (Psidium guajava L.) com potencial antioxidante para o desenvolvimento de formulação fitocosmética. Rev Bras Farmacogn 18: 387-393.

ISI-Plataforma Web of knowledge 2008. URL: http://www. periodicos.capes.gov.br.

Kojima H, Sato N, Hatano A, Ogura H 1990. Sterol glucosides from Prunella vulgaris. Phytochemistry 29: 23512355.

Lhullier C, Horta PA, Falkenberg M 2006. Avaliação de extratos de macroalgas bênticas do litoral catarinense utilizando o teste de letalidade para Artemia salina. Rev Bras Farmacogn 16: 158-163.

Lima AR, Barbosa VC, Santos Filho PR, Gouvêa CMCP 2006. Avaliação in vitro da atividade antioxidante do extrato hidroalcoólico de folhas de bardana. Rev Bras Farmacogn 16: 531-536.

Ma L, Dolphin D 1996. Stereoselective synthesis of new chlorophyll a related antioxidants isolated from marine organisms. J Org Chem 61: 2501.

Mclaughlin JL, Saizarbitori T-C, Anderson JE 1995. Tres bioensayos simples para quimicos de productos naturales. Rev Soc Venez Quim 18: 13-18.

Mensor LL, Menezes FS, Leitão GG, Reis AS, dos Santos TC, Coube CS, Leitão SG 2001. Screnning of Brazilian plant extracts for antioxidant activity by the use of DPPH free radical method. Phytother Res 15: 127130.

Meyer BN, Ferrigni NR, Putnam JE, Jacobsen LB, Nichols DE, Mclaughlin JL 1982. Brine shrimp, a convenient general bioassy for active-plant constituents. Planta Med 45: 31-34.

Mihara R, Mitsunaga T, Fukui Y, Nakai M, Yamaji N, Shibata 2004. A novel acylated quercetin tetraglycoside from oolong tea (Camelia sinensis) extracts. Tetrahedron Lett 45: 5077-5080.

Mota KSL, Pita JCLR, Estevam EC, Medeiros VM, Tavares JF, Agra MF, Diniz MFFM, Silva MS, Batista LM 2008. Evaluation of the toxicity and antiulcerogenic activity of the ethanol extract of Maytenus obtusifolia Mart. Leaves. Rev Bras Farmacogn 18: 441-446.

Neilson IC 1992. Mimosaceae (Leguminosae - Mimosoideae). Flora Malesiana. Vol. 11, Part 1. Rijksherbarium, Leiden.

Nick A, Rali T, Sticher O 1995. Biologial screening of traditional medicinal plants from Papua New Guinea. J Ethnopharmacol 49: 147-156.

Rocha FD, Pereira RC, Kaplan MAC, Teixeira VL 2007. Produtos naturais de algas marinhas e seu potencial antioxidante. Rev Bras Farmacogn 17: 631-639. 
Rodríguez M, Hasegawa M, González-Mújica F, Motta N, Castillo A, Castillo J, Zea E, Mora K, Sousa L, González A, Camejo D 2008. Antidiabetic and antiradical activities of plants from Venezuelan Amazon. Rev Bras Farmacogn 18: 331-338.

Ruiz ALTG, Magalhães EG, Magalhães AF, Faria AD, Amaral MCE, Serrano DR, Zanotti-Magalhães EM, Magalhães LA 2005. Avaliação da atividade tóxica em Artemia salina e Biomphalaria glabrata de extratos de quatro espécies do gênero Eleocharis (Cyperaceae). Rev Bras Farmacogn 15: 98-102.

SDBS 2008. Spectral Database for Organic Compounds. Acesso http://riodb01.ibase.aist.go.jp/sdbs/cgi-bin/ cre_index.cgi?lang=eng.

Shoeb M, MacManus SM, Jaspars M, Kong-Thoo-Lin P, Nahar L, Celik S, Sarker SD 2007. Bioactivity of two Turkish endemic Centaurea species, and their major constituents. Rev Bras Farmacogn 17: 155-159.

Silva TMS, Nascimento RJB, Batista MM, Agra MF, Camara CA 2007. Brine shrimp bioassay of some species of Solanum from Northestern Brazil. Rev Bras Farmacogn 17: 35-38.

Souza TJT, Apel MA, Bordignon S, Matzenbacher NI, Zuanazzi JAS, Henriques AT 2007. Composição química e atividade antioxidante do óleo volátil de Eupatorium polystachyum DC. Rev Bras Farmacogn 17: 368-372.

Vicentino ARR, Menezes FS 2007. Atividade antioxidante de tinturas vegetais, vendidas em farmácias com manipulação e indicadas para diversos tipos de doenças pela metodologia do DPPH. Rev Bras Farmacogn 17: 384-387. 